\title{
Towards Autonomous Indoor Micro VTOL
}

\section{Journal Article}

Author(s):

Bouabdallah, Samir; Murrieri, Pierpaolo; Siegwart, Roland

Publication date:

2005

Permanent link:

https://doi.org/10.3929/ethz-b-000081693

Rights / license:

In Copyright - Non-Commercial Use Permitted

Originally published in:

Autonomous Robots 18(2), https://doi.org/10.1007/s10514-005-0724-z 
Autonomous Robots 18, 171-183, 2005

\title{
Towards Autonomous Indoor Micro VTOL
}

\author{
SAMIR BOUABDALLAH \\ Autonomous Systems Lab, EPFL, CH-1015, Lausanne, Switzerland \\ samir.bouabdallah@epfl.ch \\ PIERPAOLO MURRIERI \\ Centro E. Piaggio, University of Pisa, 56126 Pisa, Italy \\ p.murrieri@ing.unipi.it \\ ROLAND SIEGWART \\ Autonomous Systems Lab, EPFL, CH-1015, Lausanne, Switzerland \\ roland.siegwart@epfl.ch
}

\begin{abstract}
Recent progress in sensor technology, data processing and integrated actuators has made the development of miniature flying robots fully possible. Micro VTOL ${ }^{1}$ systems represent a useful class of flying robots because of their strong capabilities for small-area monitoring, building exploration and intervention in hostile environments. In this paper, we emphasize the importance of the VTOL vehicle as a candidate for the high-mobility system emergence. In addition, we describe the approach that our lab ${ }^{2}$ has taken to micro VTOL evolving towards autonomy and present the mechanical design, dynamic modelling, sensing, and control of our indoor VTOL autonomous robot OS4. ${ }^{3}$
\end{abstract}

Keywords: micro VTOL, VTOL design, VTOL control, quadrotor architecture, lyapunov controller, design optimization

\section{Introduction}

Autonomous flying robots have gained enormous commercial potential during the last years. Recent developments in high density power storage, integrated miniature actuators and $\mathrm{MEMS}^{4}$ technology sensors have made autonomous miniaturized flying robots possible. This new situation has opened the way to several, complex and highly important applications for both military and civilian markets. Military applications currently represent the lion's part of the unmanned flying vehicle market, and this industrial sector is growing strongly. $\mathrm{UAVs}^{5}$ provide a significant military advantage and are routinely used in weapons plant monitoring, strategic spying, enemy territory reconnaissance and homeland defense. Depending on the flying principle and the propulsion mode, one can classify aircraft vehicles in multiple categories as shown in Fig. 1.

In the motorized heavier-than-air category, a new generation of $\mathrm{MAV}^{6}$ with a wingspan less than $15 \mathrm{~cm}$ and less than 100 grams in mass has emerged. Generally these MAVs are fully equipped with stabilization sensors in addition to miniature cameras and transmitters down-linking live video to the pilot. Military missions for MAVs are generally visual reconnaissance, situational awareness, damage assessment, surveillance, biological or chemical agent sensing, and communication relay. In addition, there are several civil applications, such as search and rescue, air sampling and field research. The Black Widow MAV from AeroVironment (Grasmeyer and Keennon) is a $15 \mathrm{~cm}$ span, 


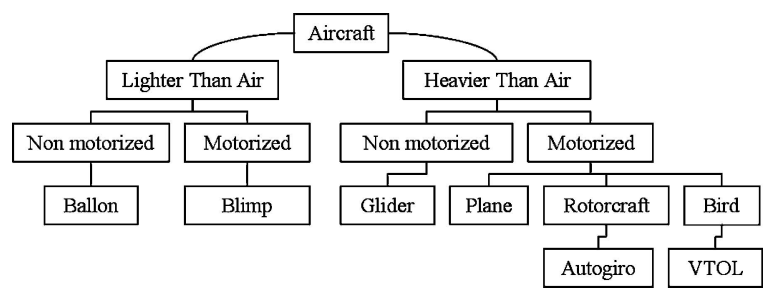

Figure 1. Aircraft general classification depending on the flying principle and the propulsion mode.

fixed-wing aircraft with an embedded color camera. It flies at $48 \mathrm{~km} / \mathrm{h}$ with an endurance of 30 minutes, and a maximum communications range of $2 \mathrm{~km}$. Bird-like MAVs seem to be the perfect solution for fast navigation in narrow spaces, obstacle avoidance in highly dynamic environments and perhaps the best approach to MAV miniaturization. Several research groups are conducting advance studies in Micromechanical Flying Insects (MFI), mainly in aerodynamics and Micromechanical systems design. The MFI project at UC Berkeley (Deng et al., 2003) uses biomimetic principles to develop a flapping wing MAV. This project represents one of the most promising endeavors towards autonomous MFIs.

The state of the art in micro helicopters is not far behind, and considerable efforts are being made, especially in control and miniaturization. Mesicopter (Kroohttp and Prinz), an ambitious project currently underway is exploring the science of millimeter and centimeter-size vehicles; in spite of unfavorable scaling laws. The project's main driving application is the deployment over large areas or planets of a huge number of micro vehicles providing atmospheric and meteorological data.

\subsection{Helicopters vs Other Flying Principles}

Compared with the other flying principles discussed above, VTOL systems have specific characteristics which allow the execution of applications that would be difficult or impossible otherwise. Table 1 gives a non-exhaustive comparison between the different flying principles from the miniaturization point of view. From this table, one can easily conclude that the VTOL systems like helicopters or blimps have an unquestionable advantage compared to the other concepts. This is thanks to their unique ability for vertical, stationary and low speed flight. These two flying concepts are the potential candidates for the high mobility systems
Table 1. Flying principles comparison focused on ability to miniaturization. $(1=\mathrm{Bad}, 2=$ Medium, $3=$ Good $)$.

\begin{tabular}{lccccc}
\hline & Airplane & Helicopter & Bird & Autogiro & Blimp \\
\hline Power cost & 2 & 1 & 1 & 2 & 3 \\
Control cost & 2 & 1 & 1 & 2 & 3 \\
Payload/volume & 3 & 2 & 2 & 2 & 1 \\
Maneuverability & 2 & 3 & 3 & 2 & 1 \\
DOF & 1 & 3 & 3 & 2 & 1 \\
Stationary flight & 1 & 3 & 2 & 2 & 3 \\
Low speed fly & 1 & 3 & 2 & 2 & 3 \\
Vulnerability & 2 & 2 & 3 & 2 & 2 \\
VTOL & 1 & 3 & 2 & 1 & 3 \\
Endurance & 2 & 1 & 2 & 1 & 3 \\
Miniaturization & 2 & 3 & 3 & 2 & 1 \\
Indoor usage & 1 & 3 & 2 & 1 & 2 \\
Total & 20 & 28 & 26 & 21 & 26 \\
\hline
\end{tabular}

emergence, as they fit the present market requirement for high mobility applications. The key advantage of blimps is the "auto-lift" and the simplicity of control, which can be essential for critical applications such as space exploration (Elfes et al., 2003) or long term navigation over cities and inhabited zones, seen in terms of miniaturization criterion. However, helicopters with different configurations probably represent currently the most promising flying concept.

\section{The OS4 Project}

The OS4 project initiated at the Autonomous Systems Laboratory (EPFL), focuses on micro VTOL vehicles evolving towards full autonomy. The project started in March 2003 with search and monitoring, indoor navigation and intervention in hostile environments as main target applications. The project goal is the design and the control of an indoor micro helicopter, capable of safe and fully autonomous stabilization and indoor navigation while transmitting high quality data.

\subsection{Our Vision of Micro VTOL Design}

Behind the actual MAV's military applications, we believe that a strong need for civilian applications potentially exists. In order to capture this potentiality, any new micro VTOL design should fit the indoor environment requirements in terms of safety, facility of use and 
respect of the environment. Our vision is to integrate actual state of the art sensing, computing, actuating and powering elements into a miniaturized VTOL vehicle. This integration should be a result of the miniaturization effect analysis and the optimization of the vehicle's elements combination.

2.1.1. Sensing the Flight. The OS4 project would not have been possible without the recent developments in inertial and vision sensor technologies. From these developments has emerged a new generation of millimeter size gyroscopes, accelerometers and cameras allowing the integration on micro VTOLs. During OS4 project we propose the use of these sensors for the configuration stabilization and indoor navigation. However, the sensing problematic in indoor MAVs is not completely solved. For example, the need for high resolution height sensors and very fast sensor interfaces remain present.

2.1.2. Computing the Flight. Carrying out our design integration and optimization effort to reality depends also on our ability to process the sensors data and compute the control algorithms in real-time, spend a minimum of power and save a maximum of place and payload. Following this goal, our approach is to integrate the new generation of tiny but powerful single board computers with high communication possibilities. Thus, the time critical configuration stabilization could easily be performed on-board while the high level controllers running on a ground station. This solution seems to be the best compromise one can do to reach maximum autonomy.

2.1.3. Actuating the Flight. Many promising new technologies of miniature motors and actuators are under development and they will certainly push farther the miniaturization limits. However, the most affordable technology available for us today is the electrical motors. In spite of the uncontestable reliability and simplicity in control of the DC brushed motors, the BLDC motors ${ }^{7}$ seem to be the best technology for micro VTOL applications as it has definitely the best power to weight ratio (including controllers) and the longest lifespan. In spite of all this advantages, small DC motors still suffer from thermal limitation which is presently one of the strongest barriers toward long endurance of micro VTOL. Thus, our aim is to find the best compromise between performance and power consumption.
2.1.4. Powering the Flight. MAV design industry is presently taking profit from the rapid technological development of high density power storage for mobile handheld devices. The best technology available for us today is Lithium-Polymer, it has around $180 \mathrm{Wh} / \mathrm{kg}$ and it allows high current discharge capacities. Following this development, the power limitation is going to be pushed away behind thermal or miniaturization limitations.

\subsection{General Design Approach}

Combining together all these new technologies following a formal optimization method is our challenge. This decision is a consequence of a several observations. For example, VTOL vehicles control could be very costly in computing time because of the system's dynamic instability. An adequate design modification probably reduces that cost and allows a stability improvement. The target applications mentioned before, especially indoor navigation, impose a strong design constraints. The robot should be compact, safe and not noisy. In most previous studies, efforts were focused either on design or on control of such systems. This approach does not permits a global evaluation of the problem, and in the case of systems difficult to control this separation could represent a handicap. Through the OS4 project, we advocate a different approach for simultaneously working on design and control of micro VTOL vehicles. This original approach makes it possible to simplify the control by design changes and vice versa as schematized in Fig. 2.

This integrated design approach is specially suited in flying robot miniaturization. Especially because downscaling is unfavorable in term of propellers efficiency and also in system stability.

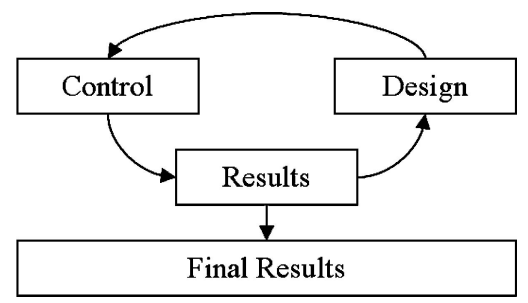

Figure 2. Our design approach in the OS4 project. In each iteration both the design and the control are modified and the results evaluated. 
A Quadrotor ${ }^{8}$ configuration vector was chosen as a starting platform for the project. This vector configuration considerably simplifies the vehicle design and intrinsically reduces the gyroscopic effects. The project started with the dynamic modelling of a Quadrotor helicopter and the development of a static method for propulsion group evaluation and optimization. In addition, a test bench was designed to experiment and tune the first controllers. However, this paper focuses on the dynamic modelling and configuration stabilization of OS4 micro VTOL.

\subsection{Quadrotor Configuration}

The Quadrotor concept has been around for a long time. The Breguet-Richet Quadrotor helicopter Gyroplane No. 1 built in 1907 is reported to have lifted into flight, and carried the pilot as high as $1.5 \mathrm{~m}$ (Leishman), The two pairs of propellers $(1,3)$ and $(2,4)$ turn in opposite directions. By varying the rotor speeds, one can change the lift forces and create motion. Thus, increasing or decreasing the four propeller's speeds together generates vertical motion. Changing the 2 and 4 propeller's speed conversely produces roll rotation coupled with lateral motion. Pitch rotation and the corresponding lateral motion result from 1 and 3 propeller's speed conversely modified as described in Fig. 3. Yaw rotation is more subtle, as it results from the difference in the counter-torque between each pair of propellers.

2.3.1. Advantages and Drawbacks. Although disadvantages, such as space and energy requirements for the Quadrotor, spring more quickly to mind than the system's advantages, this concept offers a better payload and is potentially simpler to build and to control. This could be a decisive advantage. Table 2 gives a rapid idea about Quadrotor's advantages and drawbacks.

Table 2. Quadrotor main advantages \& drawbacks.

\begin{tabular}{lc}
\hline Advantages & Drawbacks \\
\hline Rotor mechanics simplification & Weight augmentation \\
Payload augmentation & High energy consumption \\
Gyroscopic effects reduction & \\
\hline
\end{tabular}

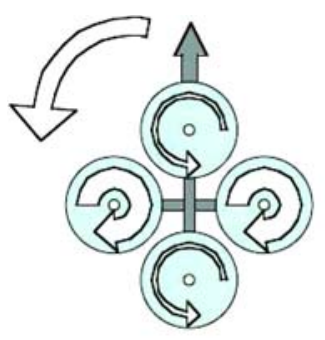

Rotate left

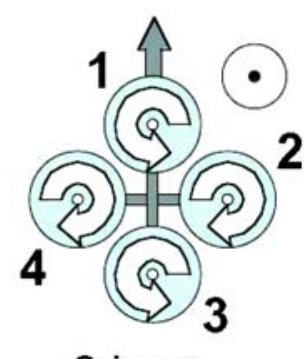

Going up

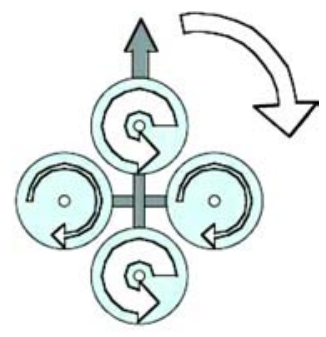

Rotate right

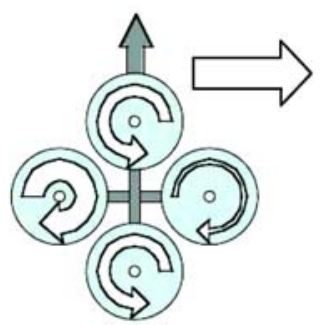

Move right
Figure 3. Quadrotor concept motion description, the arrow width is proportional to propeller rotational speed.

\subsection{Quadrotor Dynamic Modelling}

The first step before control development is an adequate dynamic system modelling (Padfield, 1996). Especially for lightweight systems, the dynamic model ideally includes the gyroscopic effects resulting from both the rigid body rotation in space and the four propeller's rotation. This effect has been often neglected in previous works. However, the main effects acting on a helicopter are described briefly in Table 3.

Let us consider earth fixed frame $E$ and body fixed frame $B$, as seen in Fig. 4 . The center of mass and the

Table 3. Main physical effects acting on a helicopter (see table below for definitions).

\begin{tabular}{llc}
\hline Effect & \multicolumn{1}{c}{ Source } & Formulation \\
\hline Aerodynamic effects & $\begin{array}{l}\text { - Propeller rotation } \\
\text { - Blades flapping } \\
\text { Inertial counter torques }\end{array}$ & $\begin{array}{l}\text { - Change in propeller } \\
\text { rotation speed }\end{array}$ \\
Gravity effect & $J \dot{\Omega}$ \\
Gyroscopic effects & $\begin{array}{l}\text { - Center of mass position } \\
\text { - Change in orientation } \\
\text { of the rigid body } \\
\text { - Change in orientation } \\
\text { Friction }\end{array}$ & $J \theta \psi$ \\
\hline
\end{tabular}




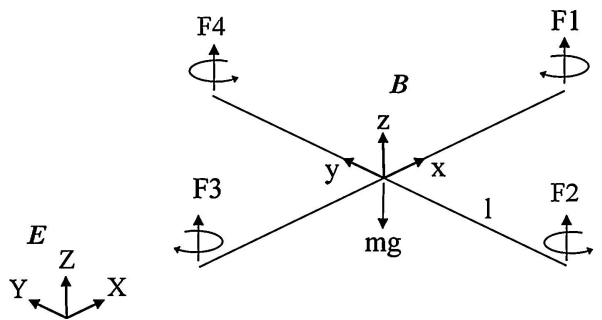

Figure 4. Quadrotor configuration frame system with a body fixed frame $B$ and the inertial frame $E$.

body fixed frame origin are assumed to coincide. Using Euler angles parametrization, the airframe orientation in space is given by a rotation $R$ from $B$ to $E$, where $R \in S O 3$ is the rotation matrix.

$R=\left(\begin{array}{ccc}c \psi c \theta & s \phi s \theta c \psi-c \phi s \psi & c \phi s \theta c \psi+s \phi s \psi \\ s \theta s \psi & s \phi s \theta s \psi+c \theta c \psi & c \phi s \theta s \psi \\ -s \theta & s \phi c \theta & c \phi c \theta\end{array}\right)$

The dynamics of a rigid body under external forces applied to the center of mass and expressed in the body fixed frame as shown in Sastry (1994) and Chriette (2001) are in Newton-Euler formalism:

$$
\left[\begin{array}{cc}
m I_{3 \times 3} & 0 \\
0 & I
\end{array}\right]\left[\begin{array}{c}
\dot{V} \\
\dot{\omega}
\end{array}\right]+\left[\begin{array}{c}
\omega \times m V \\
\omega \times I \omega
\end{array}\right]=\left[\begin{array}{c}
F \\
\tau
\end{array}\right]
$$

Where $I \in \mathfrak{R}^{(3 \times 3)}$ the inertia matrix, $V$ the body linear speed vector and $\omega$ the body angular speed.

Using the frame system (4).

The equations of motion for the helicopter (OlfatiSaber, 2001) can be written as:

$$
\left\{\begin{array}{l}
\dot{\zeta}=v \\
m \dot{v}=R F_{b} \\
\dot{R}=R \hat{\omega} \\
J \dot{\omega}=-\omega \times J \omega+\tau_{b}
\end{array}\right.
$$

The first-level approximate model (4) of the Quadrotor can be rewritten as:

$$
\left\{\begin{array}{l}
\dot{\zeta}=v \\
\dot{v}=-g e_{3}+R_{e_{3}}\left(\frac{b}{m} \sum \Omega_{i}^{2}\right) \\
\dot{R}=R \hat{\omega} \\
I \dot{\omega}=-\omega \times I \omega-\sum J_{r}\left(\omega \times e_{3}\right) \Omega_{i}+\tau_{b}
\end{array}\right.
$$

where:

\begin{tabular}{ll}
\hline Symbol & \multicolumn{1}{c}{ Definition } \\
\hline$\zeta$ & position vector \\
$\nu$ & speed vector (expressed in $E$ ) \\
$R$ & rotation matrix \\
$\hat{\omega}$ & skew symmetric matrix \\
$\phi$ & roll angle \\
$\theta$ & pitch angle \\
$\psi$ & yaw angle \\
$\Omega$ & rotor speed \\
$I_{x, y, z}$ & body inertia \\
$J_{r}$ & rotor inertia \\
$F_{b}$ & forces on airframe body \\
$\tau_{b}$ & torques on airframe body \\
$b$ & thrust factor \\
$d$ & drag factor \\
$l$ & lever \\
$e 1,2,3$ & standard basis in $R^{3}$ \\
$g$ & acceleration due to gravity \\
\hline
\end{tabular}

The torque applied on the vehicle's body along an axis is the difference between the torque generated by each propeller on the other axis.

$$
\tau_{b}=\left(\begin{array}{c}
l b\left(\Omega_{4}^{2}-\Omega_{2}^{2}\right) \\
l b\left(\Omega_{3}^{2}-\Omega_{1}^{2}\right) \\
d\left(\Omega_{2}^{2}+\Omega_{4}^{2}-\Omega_{1}^{2}-\Omega_{3}^{2}\right)
\end{array}\right)
$$

The full Quadrotor dynamic model with the $x, y, z$ motions as a consequence of a pitch or roll rotation is in (6). The friction is neglected in all vehicle motions, except the yaw motion.

$$
\left\{\begin{array}{l}
\ddot{x}=(\cos \phi \sin \theta \cos \psi+\sin \phi \sin \psi) \frac{1}{m} U_{1} \\
\ddot{y}=(\cos \phi \sin \theta \sin \psi-\sin \phi \cos \psi) \frac{1}{m} U_{1} \\
\ddot{z}=-g+(\cos \phi \cos \theta) \frac{1}{m} U_{1} \\
\ddot{\phi}=\dot{\theta} \dot{\psi}\left(\frac{I_{y}-I_{z}}{I_{x}}\right)-\frac{J_{r}}{I_{x}} \dot{\theta} \Omega+\frac{l}{I_{x}} U_{2} \\
\ddot{\theta}=\dot{\phi} \dot{\psi}\left(\frac{I_{z}-I_{x}}{I_{y}}\right)+\frac{J_{r}}{I_{y}} \dot{\phi} \Omega+\frac{l}{I_{y}} U_{3} \\
\ddot{\psi}=\dot{\phi} \dot{\theta}\left(\frac{I_{x}-I_{y}}{I_{z}}\right)+\frac{1}{I_{z}} U_{4}
\end{array}\right.
$$


Assuming $U_{1}, U_{2}, U_{3}, U_{4}$ as the system inputs and $\Omega$ as a disturbance, we obtain:

$$
\left\{\begin{array}{l}
U_{1}=b\left(\Omega_{1}^{2}+\Omega_{2}^{2}+\Omega_{3}^{2}+\Omega_{4}^{2}\right) \\
U_{2}=b\left(\Omega_{4}^{2}-\Omega_{2}^{2}\right) \\
U_{3}=b\left(\Omega_{3}^{2}-\Omega_{1}^{2}\right) \\
U_{4}=d\left(\Omega_{2}^{2}+\Omega_{4}^{2}-\Omega_{1}^{2}-\Omega_{3}^{2}\right) \\
\Omega=\Omega_{2}+\Omega_{4}-\Omega_{1}-\Omega_{3}
\end{array}\right.
$$

\subsection{Rotor Dynamics}

The rotors are driven by DC-motors with the well known equations (Jucker, 1974):

$$
\left\{\begin{array}{l}
L \frac{d i}{d t}=u-R i-k_{e} \omega_{m} \\
J \frac{d \omega_{m}}{d t}=\tau_{m}-\tau_{d}
\end{array}\right.
$$

As we use a small motor with a very low inductance, the second order DC-motor dynamics may be approximated by:

$$
J \frac{d \omega_{m}}{d t}=-\frac{k_{m}^{2}}{R} \omega_{m}-\tau_{d}+\frac{k_{m}}{R} u
$$

By introducing the propeller and the gearbox models, the Eq. (9) may be rewritten:

$$
\left\{\begin{array}{l}
\dot{\omega}_{m}=-\frac{1}{\tau} \omega_{m}-\frac{d}{\eta r^{3} J_{t}} \omega_{m}^{2}+\frac{1}{k_{m} \tau} u \text { with : } \\
\frac{1}{\tau}=\frac{k_{m}^{2}}{R J_{t}}
\end{array}\right.
$$

The Eq. (10) can be linearized around an operation point $\dot{w}_{0}$ to the form $\dot{w}_{m}=-A w_{m}+B u+C$ with:

$$
A=\left(\frac{1}{\tau}+\frac{2 d w_{0}}{\eta r^{3} J_{t}}\right), \quad B=\left(\frac{1}{k_{m} \tau}\right), \quad C=\frac{d \omega_{0}^{2}}{\eta r^{3} J_{t}}
$$

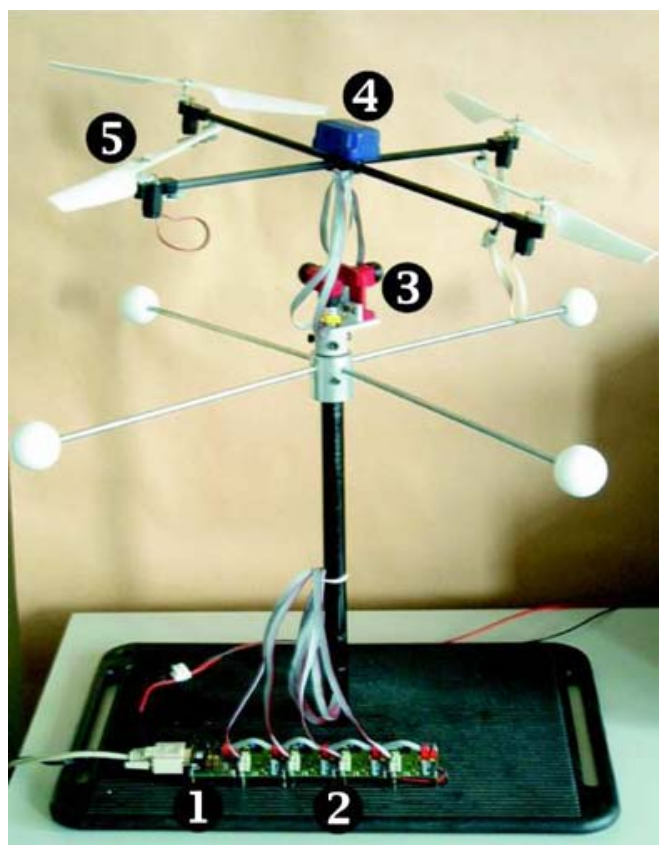

Figure 5. OS4 test bench for stabilization strategies testing, 3DOF are locked using the $3 \mathrm{D}$ joint, the cross is made with carbon rods and the overall weight is about $7 \mathrm{~kg}$.

\subsection{OS4 Test Bench Design}

The development of a control system for a flying robot requires the development of an adequate test bench at least for the preliminary experiments. This can help lock some number of degrees of freedom in order to reduce control complexity and to avoid system damage and ensure security of the persons working around. In Fig. 5:

(1) RS232 to I2C translator.

(2) Motor modules (04).

(3) 3D captured universal joint.

(4) Micro IMU 9 (xsens MT9-B)

(5) Propulsion group.

The Fig. 6, shows the test bench main component's block diagram.

Through a standard RS232 port one can send orders from the controller (PC @ $450 \mathrm{Mhz}$ ) to the system. The RS232 to I2C module translates the serial RS232 signals to the I2C bus motor modules. These modules integers a PID regulator on a PIC16F876 microcontroller and are capable of open or closed loop operation in position, speed or torque control. 


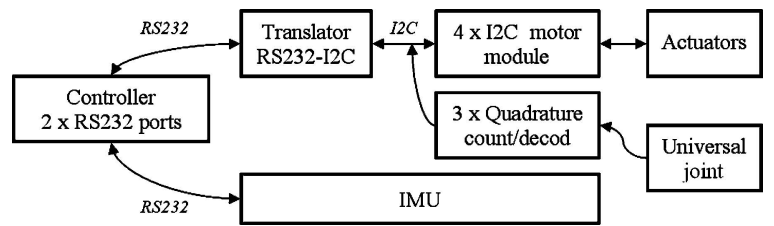

Figure 6. OS4 test bench block diagram.

The MT9-B sensor ${ }^{10}$ estimates with a kalman filter the $3 \mathrm{D}$ orientation data and gives the calibrated data of angular velocity and acceleration. It weights about $33 \mathrm{~g}$ and communicates at $115 \mathrm{kbps}$. The captured motion from the $3 \mathrm{D}$ universal joint ${ }^{11}$ can be decoded to extract absolute orientation information, thanks to the micro optical encoders in each axis. The vehicle is thus lightweight, about $235 \mathrm{~g}$ for all the system. The OS4 test bench has 4 propulsion groups, each composed of a $29 \mathrm{~g}$ motor $^{12}$ including magnetic encoders, a $6 \mathrm{~g}$ gearbox and a $6 \mathrm{~g}$ propeller. To design the propulsion group, a test, evaluation and comparison method was developed.

\subsubsection{Propulsion Group Evaluation and Design Pro-}

cedure. Finding the highest thrust to weight ratio is one of the most important challenges in micro VTOL design (Nicoud and Zufferey, 2002). Our approach was firstly to specify the application requirements in terms of thrust, energy and overload allowed. Secondly it was to build a propeller and motor data bank and then find the best combination. Finally we compare the results to the requirements, see Fig. 7. For the propeller databank, we use a specific test bench to extract thrust and drag coefficients through experiments where we measure tension, current, thrust and rotational speed. As seen in Fig. 8, a compact and lightweight plastic made gear-box was designed, it weights approximately $6 \mathrm{~g}$ and has about $90 \%$ efficiency.

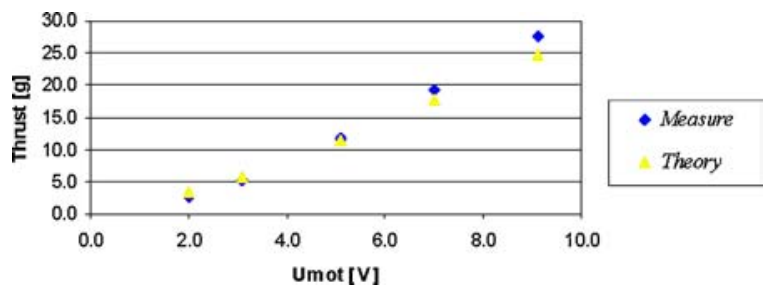

Figure 7. Theoretical and measured thrust comparison using the evaluation tool.

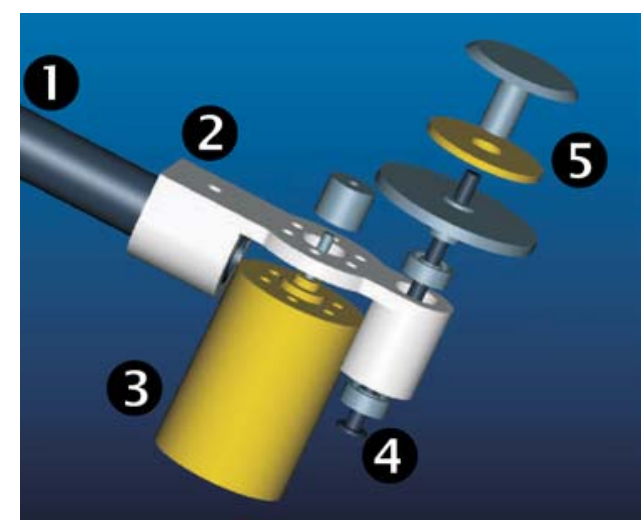

Figure 8. Exploded picture of the propulsion group CAD model. (1) Carbon rod, (2) Main part, (3) Motor, (4) Axis and micro Ball bearings, (5) Propeller fixing.

Designing a flying robot is an iterative process and one has to fix starting conditions. For our development, we have chosen to start from the vehicle size approximate determination which allows the propeller selection from the data base according to it's size. Using the evaluation tool, one can easily select the appropriate motor. Finally we use the well known motor equations to determine the optimal reduction ratio for our propulsion group.

From the Fig. 9 one can easily select the best motors for his application. The tool includes a DC-motor thermal model to evaluates the working time under the applied load, see Fig. 10.

$$
\tau_{t h} \frac{d T}{d t}+\left(T-T_{1}\right)=R_{t h} \times P_{v}
$$

The warming differential Eq. (12) accepts (13) as a solution if $P_{v}$ is time constant (Jucker, 1974).

$$
T=T_{1}+R_{t h} \times P_{v}\left(1-e^{-\frac{t}{\tau_{t h}}}\right)
$$

With:

\begin{tabular}{ll}
\hline Symbol & \multicolumn{1}{c}{ Definition } \\
\hline$\tau_{t h}$ & thermal time constant. \\
$R_{t h}$ & thermal resistance. \\
$T$ & rotor temperature. \\
$T_{1}$ & Stator temperature \\
$P_{v}$ & dissipated power \\
\hline
\end{tabular}




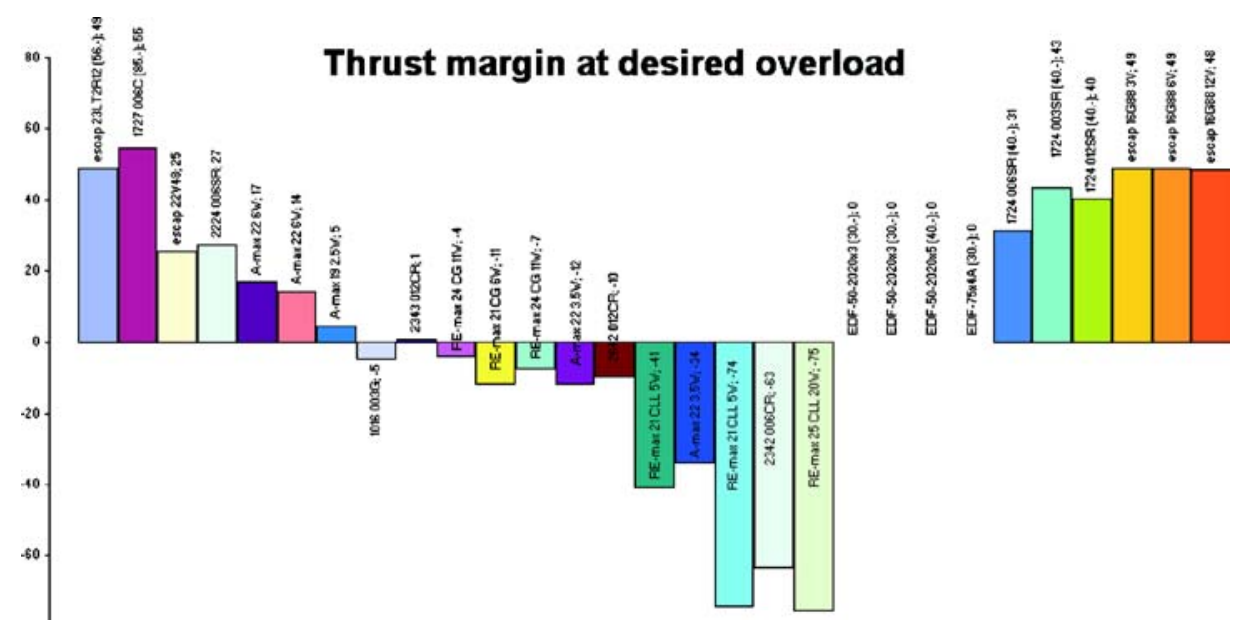

Figure 9. Motor comparison for a specific requirement.

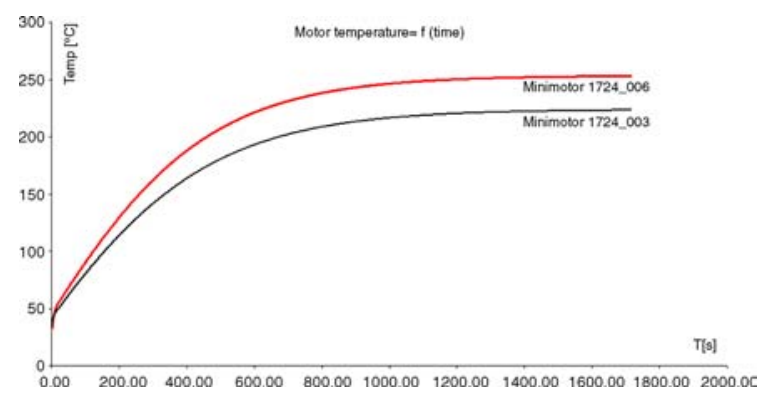

Figure 10. Theoretical time-evolution of rotor temperature in two different motors (in our application), the propeller reduces the motor temperature in practice.

\section{Control of the VTOL System}

The model (6), developed in the previous sections, can be rewritten in a state-space form $\dot{X}=f(X, U)$ by introducing $X=\left(x_{1} \ldots x_{12}\right)^{T} \in \mathfrak{R}^{12}$ as state vector of the system as follows:

$$
\begin{aligned}
& x_{1}=x \\
& x_{2}=\dot{x}_{1}=\dot{x} \\
& x_{3}=y \\
& x_{4}=\dot{x}_{3}=\dot{y} \\
& x_{5}=z \\
& x_{6}=\dot{x}_{5}=\dot{z} \\
& x_{7}=\phi \\
& x_{8}=\dot{x}_{7}=\dot{\phi}
\end{aligned}
$$

$$
\begin{aligned}
x_{9} & =\theta \\
x_{10} & =\dot{x}_{9}=\dot{\theta} \\
x_{11} & =\psi \\
x_{12} & =\dot{x}_{11}=\dot{\psi}
\end{aligned}
$$

From (14) and (6) we obtain:

$$
f(X, U)=\left(\begin{array}{c}
x_{2} \\
\left(\cos x_{7} \sin x_{9} \cos x_{11}+\sin x_{7} \sin x_{11}\right) \frac{U_{1}}{m} \\
x_{4} \\
\left(\cos x_{7} \sin x_{9} \sin x_{11}-\sin x_{7} \cos x_{11}\right) \frac{U_{1}}{m} \\
x_{6} \\
-g+\left(\cos x_{7} \cos x_{9}\right) \frac{1}{m} U_{1} \\
x_{8} \\
x_{12} x_{10}\left(\frac{I_{y}-I_{z}}{I_{x}}\right)-\frac{J_{R}}{I_{x}} x_{10} \Omega+\frac{l}{I_{x}} U_{2} \\
x_{10} \\
x_{12} x_{8}\left(\frac{I_{z}-I_{x}}{I_{y}}\right)+\frac{J_{R}}{I_{y}} x_{8} \Omega+\frac{l}{I_{y}} U_{3} \\
x_{10} x_{8}\left(\frac{I_{x}-I_{y}}{I_{z}}\right)+\frac{l}{I_{z}} U_{4}
\end{array}\right)
$$

It is worthwhile to note inside the dynamic of the latter system how the angles and their time derivatives do not depend on translation components; on the other hand the translations depend on angle (and not on angular 


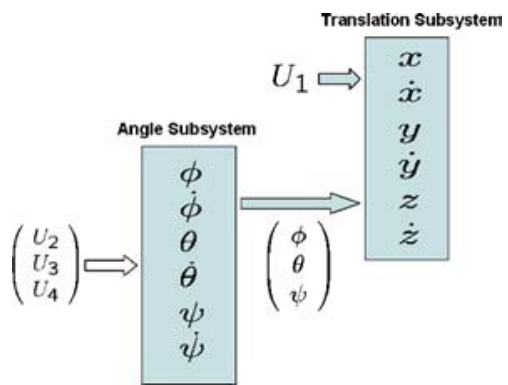

Figure 11. Connection of the two ideal subsystems of which is composed the overall dynamical system described by mapping (15). From the angular rotations subsystem the roll, pitch and yaw are obtained and become with $U_{1}$ inputs for the following translation subsystem.

velocities). We can ideally imagine the overall system described by (15) as constituted by two subsystems, the angular rotations and the linear translations, see Fig. 11. The angular rotations subsystem has as state the restriction $X_{\alpha}$ of $X$ to the last 6 components which regard the roll, pitch, yaw and their time derivative. The dynamics of these variables are described by $f_{\alpha}(X, U)$ which corresponds to the last 6 components of the mapping (15). Note that the mapping $f_{\alpha}(X, U)$ is function only of $X_{\alpha}$ and of $\left(U_{2}, U_{3}, U_{4}\right)^{T}$, and does not depend on translation components. On the other hand, the translations subsystem (with state $X_{\Delta}$ ) regards the first 6 element of the state $X$, which are the $x, y, z$ and their time derivative; in this case too the dynamics are described by the first 6 rows $f_{\Delta}(X, U)$ of the mapping (15). Conversely from the previous case, the translations subsystem mapping $f_{\Delta}(X, U)$ is not independent of the angle variables but depends only on roll, pitch and yaw and not on their time derivative.

\subsection{Control of the Angular Rotations Subsystem}

Due to its complete independence from the other subsystem, it is interesting to consider first the control of the angular rotations subsystem. In particular, in this subsection we consider the stabilization of the OS4 angles in a particular configuration $X_{\alpha}^{d}=$ $\left(x_{7}^{d}, 0, x_{9}^{d}, 0, x_{11}^{d}, 0\right)^{T}$.

Let us consider the Lyapunov Function $V\left(X_{\alpha}\right)$ which is $C^{1}$ and positive defined around the desired position $X_{\alpha}^{d}$ :

$\frac{\left(x_{7}-x_{7}^{d}\right)^{2}+x_{8}^{2}+\left(x_{9}-x_{9}^{d}\right)^{2}+x_{10}^{2}+\left(x_{11}-x_{11}^{d}\right)^{2}+x_{12}^{2}}{2}$.
The time derivative of (16), $\dot{V}=(\nabla V)^{T} f_{\alpha}$, in the case of a perfect cross VTOL $\left(I_{x}=I_{y}\right)$ is drastically reduced to:

$$
\begin{aligned}
\dot{V}= & \left(x_{7}-x_{7}^{d}\right) x_{8}+x_{8} \frac{l}{I_{x}} U_{2}+\left(x_{9}-x_{9}^{d}\right) x_{10} \\
& +x_{10} \frac{l}{I_{y}} U_{3}+\left(x_{11}-x_{11}^{d}\right) x_{12}+x_{12} \frac{l}{I_{z}} U_{4}
\end{aligned}
$$

equation in which does not appear the disturbance term with $\Omega$. By simply choosing:

$$
\begin{aligned}
& U_{2}=-\frac{I_{x}}{l}\left(x_{7}-x_{7}^{d}\right)-k_{1} x_{8} \\
& U_{3}=-\frac{I_{y}}{l}\left(x_{9}-x_{9}^{d}\right)-k_{2} x_{10} \\
& U_{4}=-I_{z}\left(x_{11}-x_{11}^{d}\right)-k_{3} x_{12},
\end{aligned}
$$

with $k_{1}, k_{2}$ and $k_{3}$ positive constants, we obtain for (17):

$$
\dot{V}=-x_{8}^{2} \frac{l k_{1}}{I_{x}}-x_{10}^{2} \frac{l k_{2}}{I_{y}}-x_{12}^{2} \frac{k_{3}}{I_{z}},
$$

which is only negative semi-defined. By Lyapunov theorem (Arimoto, 1996) is now ensured the simple stability for equilibrium. By Lasalle invariance theorem we can ensure also that starting from a level curve of the Lyapunov function defined in (16) where $V\left(X_{\alpha}\right)$ is constant, the state evolution is constrained inside the region bounded by the level curve. This is very useful when trying to avoid particular configuration; it is simply necessary to start with a level curve not containing these points and apply the previous defined controls. We can also ensure the asymptotic stability by applying the Lasalle theorem because the maximum invariance set of (angular rotations) subsystem under control (18) contained in the set $S=\left\{X_{\alpha}^{S} \in \Re^{6}:\left.\dot{V}\right|_{X_{\alpha}^{S}}=0\right\}$ is restricted only to the equilibrium point.

By the latter consideration we can ensure an asymptotical stability starting from a point in a set around the equilibrium. To ensure the global stability it is sufficient that the $\lim _{\left|X_{\alpha}\right| \rightarrow \infty} V\left(X_{\alpha}\right)=\infty$, which is our case.

\subsection{Height Controller}

Let us consider the simple task for the VTOL to hover at a particular height $z=z^{d}$. The dynamic of the height 
is described by lines 5 and 6 of system (15), that is:

$$
\left(\begin{array}{c}
\dot{x}_{5} \\
\dot{x}_{6}
\end{array}\right)=\left(\begin{array}{c}
x_{6} \\
-g+\cos x_{7} \cos x_{9} \frac{U_{1}}{m}
\end{array}\right) .
$$

By the previous considerations in 3.1, we ensure that starting from an initial condition where $V\left(X_{\alpha}\right)<\frac{\pi}{2}$, the angles and their velocities are constrained in this hypersphere of $\Re^{6}$. In this case $\cos x_{7} \cos x_{9} \neq 0$ during all the trajectories of the system under previous control law. If the latter condition is satisfied we can linearize system (20) by simply compensating the weight force by $U_{1}=\frac{m g}{\cos x_{7} \cos x_{9}}+\frac{m U_{1}}{\cos x_{7} \cos x_{9}}$, where $\hat{U}_{1}$ is an additional term. By the latter law (20) becomes:

$$
\left(\begin{array}{c}
\dot{x}_{5} \\
\dot{x}_{6}
\end{array}\right)=\left(\begin{array}{c}
x_{6} \\
\hat{U}_{1}
\end{array}\right),
$$

By a simple state-space linear stabilization law $\hat{U}_{1}=$ $k_{4} x_{5}+k_{5} x_{6}$ we can stabilize the height by placing the poles of the subsystem in every position in the complex left half plane.

\section{Simulations}

Before implementation on the real system, we performed several simulations on Matlab. ${ }^{13}$ The controller's task was to stabilize the height while compensating the initial error on the roll, the pitch and the yaw angles.

The real system suffers from undesired but unavoidable delays and actuator saturation. The delays are mainly due to RS232 communications and the actuator time constant. To emulate this lacks, two Simulink discrete step delay blocks have been introduced in the feedback loop and on the actuators. Saturation level depends on the chosen actuator. The motors work in our application with a maximum angular velocity of $600 \mathrm{rad} / \mathrm{sec}$; a saturation block has been placed between the controller and the actuator. Finally, the overall system has been simulated at $30 \mathrm{~Hz}$ using a discrete time solver in order to model the behavior of the digital controller. In the first simulation, the system starts with an initial state $X_{0}=\left(0,0,0,0,0,0, \frac{\pi}{10}, 0,-\frac{\pi}{10}, 0, \frac{\pi}{10}, 0\right)^{T}$. We want to reach the final height of 2 meters with all the angles at zero. In Fig. 12 we can see the evolution of the three angles and of the height variable during the 50 seconds of simulation. In the second simulation, see Fig. 13,
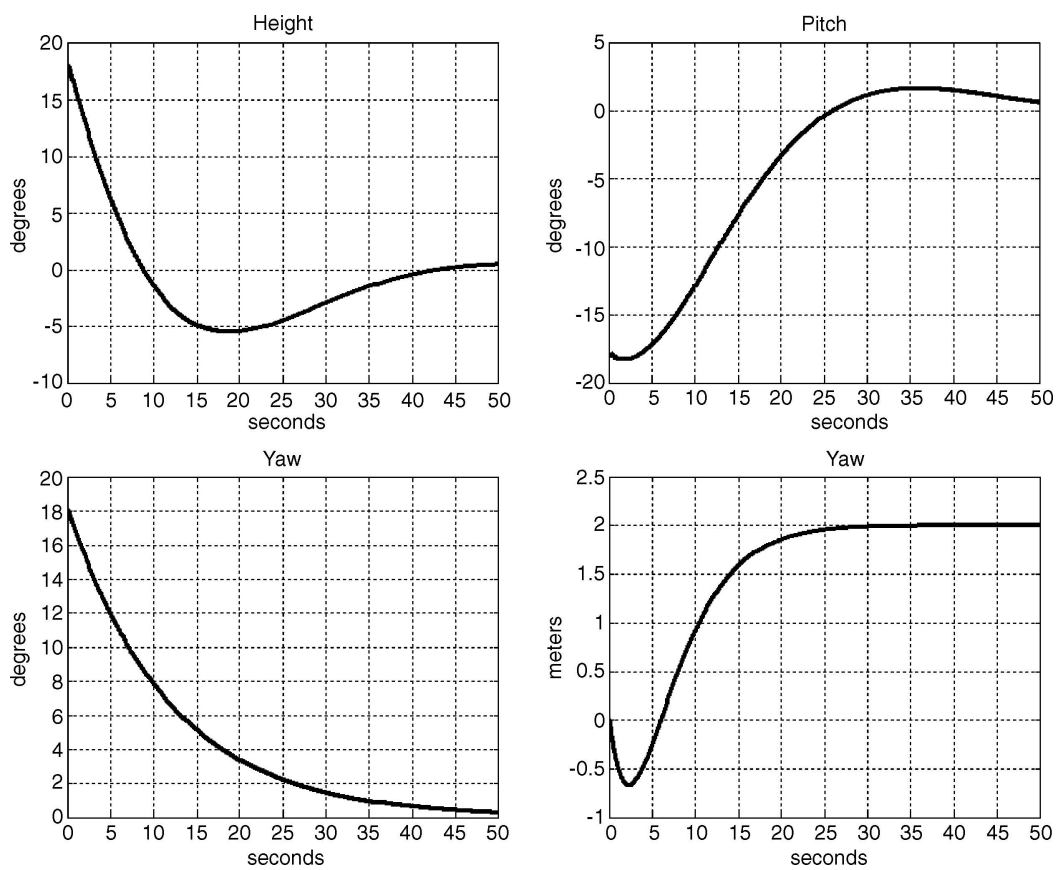

Figure 12. First Simulation: the system has to correct the errors on the angles and to reach 2 meters height. 

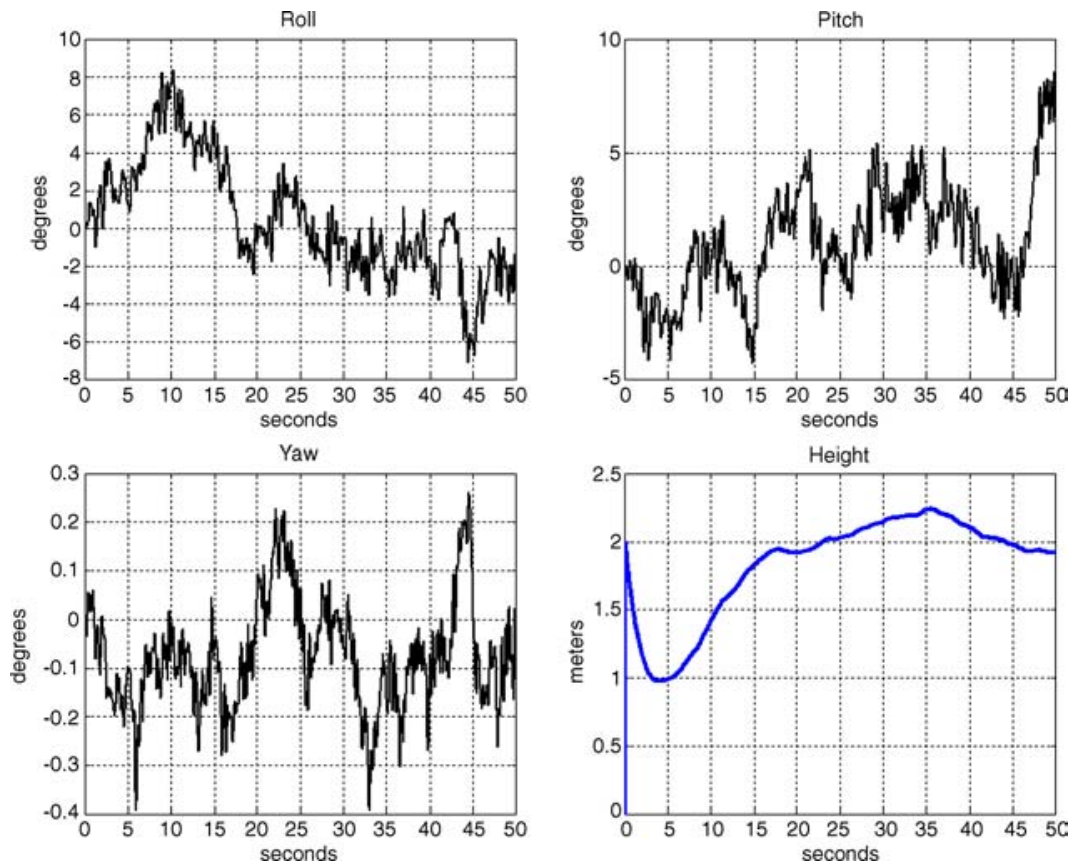

Figure 13. Second Simulation: the system has to hover and maintain the height of 2 meters although the noise on the actuators.

the task is to hover although an added normal gaussian noise of variance $4 \mathrm{rad}^{2} / \mathrm{sec}^{2}$ on each angular velocity. The height is taken with an added zero mean error.

\subsection{D Simulator}

Full autonomy from take-off to landing is possible through a combination of several control modes which are in general not developed at the same time. Testing a non complete and not validated control strategy on real systems could be a sensitive mission, the probable material damage and the potential danger on persons imposes an important simulation step. A 3D simulator was developed using $\mathrm{ODE}^{14}$ library under webots (see Fig. 14) ${ }^{15}$ For the tests only simple preliminary control lows (PID) were implemented and tested.

\section{Experiments}

In order to validate the control law developed in the previous section, we implemented the controller and we performed several experiments on the real system. The task was to control the vehicle orientation thus, the roll, the pitch and the yaw angles were controlled, see Fig. 15. While the height was fixed by the test bench.

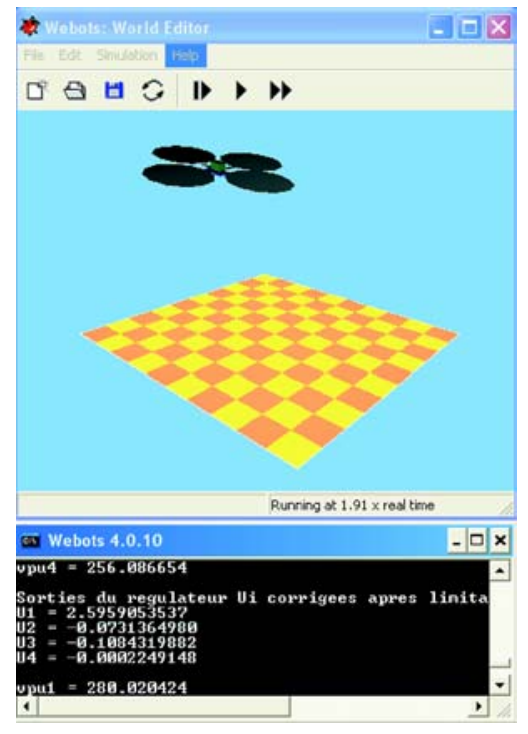

Figure 14. 3D simulation of a Quadrotor on webots already permits the test of the controller under disturbances

Therefore, for these simulations and experiments we used the same algorithms but the test conditions are slightly different namely the fixed height and the limited starting angles. 

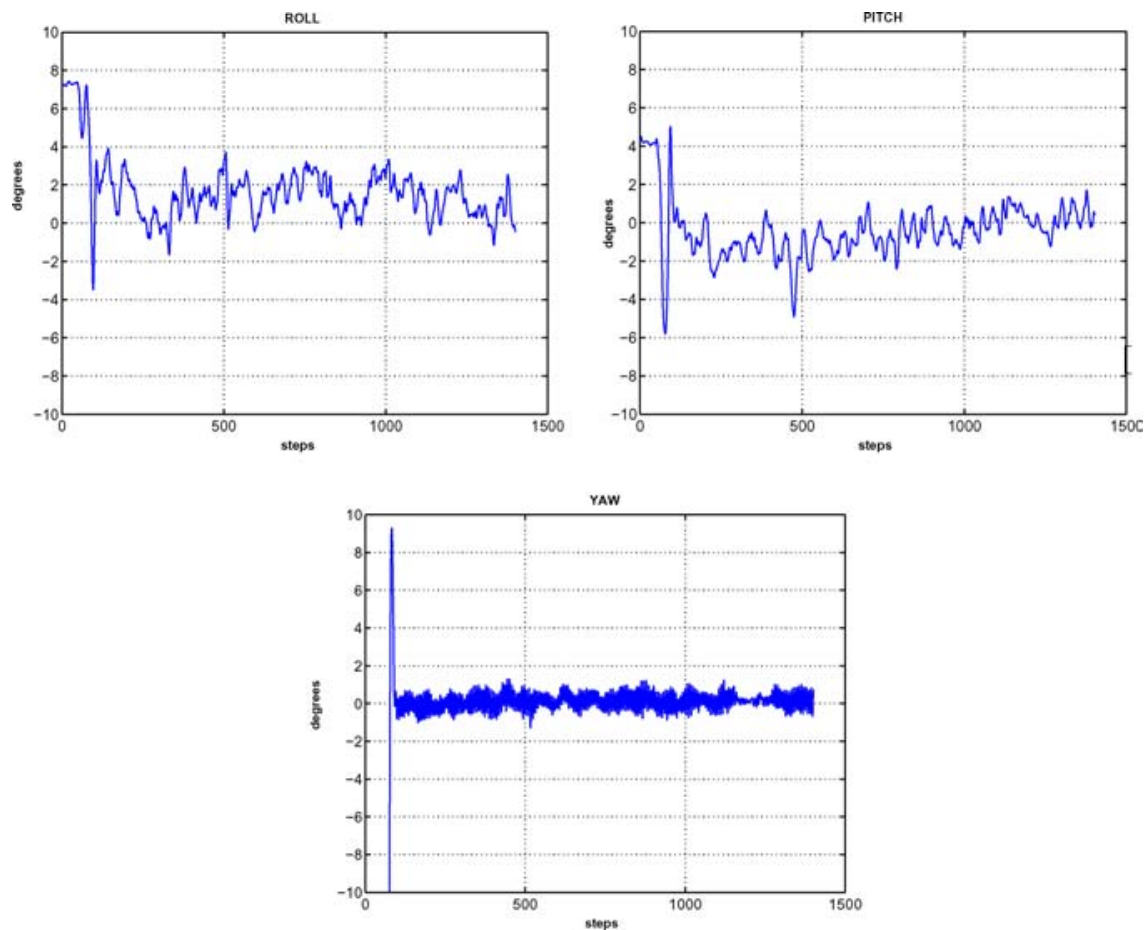

Figure 15. Experiments: the controller has to stabilize the system by maintaining the roll, pitch and yaw angels to zero.

In spite of the test bench limitations in term of delays and errors introduced by the tethering system, the experimental results obtained show that the vehicle orientation was well controlled during the 50 seconds (1500 steps@30 Hz). However, the vibrations and the noise produced by the " 04 " motors have a direct influence on the sensor data thus, a small drift was observed after a relatively long experiment especially on the yaw angle.

\section{Conclusion and Future Work}

In this paper, we presented a survey of existing flying micro-vehicles and proposed micro VTOL systems as a serious candidates for fully autonomous indoor inspection robots. We introduced the OS4 project, enumerate the advantages of the Quadrotor configuration and discussed the undergoing developments of flying robots at our lab. These includes dynamic modelling, autonomous control, vehicle design and optimization. The positive results obtained in this preliminary work towards autonomous micro VTOL reinforce our conviction that these systems have a real potential as highmobility systems. Our next goal is to enhance the control with position controller and to develop a fully au- tonomous vehicle. Then we will mainly concentrate on autonomous navigation with limited perceptual and computation resources.

\section{Acknowledgment}

The authors would like to thank Jean-Christophe Zufferey for fruitful discussions and advices on flying robots, Georges Perrenoud for the realization of the test bench mechanical components and all the students who worked or are working on this project.

\section{Notes}

1. Vertical Take-Off and Landing.

2. Autonomous Systems Lab.

3. Omnidirectional Stationary Flying OUtstretched Robot.

4. Micro Electromechanical Systems.

5. Unmanned Aerial Vehicles.

6. Micro Aerial Vehicle.

7. BrushLess Direct-Current.

8. Four propellers in cross configuration.

9. Inertial Measurements Unit.

10. www.xsens.com

11. www.forcedimension.com.

12. 1724 motor from: www.minimotor.ch. 
13. www.mathworks.com.

14. Open Dynamics Engine.

15. www.cyberbotics.com.

\section{References}

Altug, E., Ostrowski, J.P., and Mahony, R.E. 2002. Control of a quadrotor helicopter using visual feedback. In International Conference on Robotics and Automation, Washington, USA.

Arimoto, S. 1996. Control Theory of Non-linear Mechanical Systems. Oxford Science Publications.

Chriette, A. 2001. Contribution à la commande et à la modélisation des hélicoptères: Asservissement visuel et commande adaptative. Phd Thesis.

Deng, X., Schenato, L., and Sastry, S.S. 2003. Attitude Control for a Micromechanical Flying Insect Including Thorax and Sensor Models ICRA 2003, Teipei, Taiwan.

Elfes, A., Bueno, S.S., et al. 2003. Robotic Airship for Exploration of planetary Bodies with an Atmosphere Autonomy Challenges. Autonomous Robots Journal: Kluwer Academic Publishers.

Gäfvert, M. 2001. Modelling of the ETH helicopter. Department of Automatic control, Lund Institute of Technology, $\mathrm{CH}$.

Gessow, A. and Myers, G. 1967. Aerodynamics of the Helicopter, third edition. Frederick Ungar Publishing Co, New York.

Grasmeyer, J. and Keennon, M.T. Development of the Black Widow Micro Air Vehicle. AeroVironment, Simi Valley, CA 93063.

Hauser, J., Sastry, S., and Meyer, G. 1992. Nonlinear control design for slightly non-minimum phase systems: Application to V/STOL aircraft. Automatica, 28(04):665-679.

Jucker, E. 1974. Equations fondamentales des micromoteurs courant continu avec rotor sans fer. Bulletin technique Portescap, La Chaud-de-Fonds.

Kroohttp, I. and Prinz, F.B. The Mesicopter: A Meso-Scale Flight Vehicle. http://aero.stanford.edu/mesicopter/.

Leishman, J.G. The Breguet-Richet Quad-Rotor Helicopter of 1907. http://www.enae.umd.edu/AGRC/Aero/Breguet.pdf.

Martin, P., Devasia, S., and Paden, B. 1996. A different look at output tracking: Control of a VTOL aircraft. Automatica, 32(01):101107.

Nicoud, J.D. and Zufferey, J.C. 2002. Towards indoor flying robots. In Conference on Intelligent Robots and Systems, Lausanne, Switzerland.

Olfati-Saber, R. 2001. Nonlinear control of underactuated mechanical systems with application to robotics and aerospace vehicles. Phd thesis, Department of Electrical Engineering and Computer Science, MIT.

Padfield, D. 1996. Helicopter Flight Dynamics. Blackwell Science: Oxford.

Pounds, P. and Mahony, R. 2002. Design of a Four-Rotor Aerial Robot. Australasian Conference on Robotics and Automation, Auckland, Australia.

Sastry, S. 1994. A Mathematical Introduction to Robotic Manipulation. Boca Raton, FL.

Shim, H., Koo, T.J., Hoffmann, F., and Sastry, S. 1998. A comprehensive study of control design for an autonomous helicopter. In Conference on Decision and Control, Florida.

Suter, D., Hamel, T., and Mahony, R. 2002. Visual servo control using homography estimation for the stabilization of an X4-fyler. In Conference on Decision and Control, Nevada.

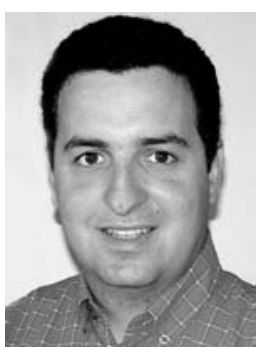

Samir Bouabdallah is research assistant and Ph.D. student at the Autonomous Systems Lab (ASL) at the Swiss Federal Institute of Technology, Lausanne, (EPFL). He got his Masters in Electrical Engineer from Abu Bakr Belkaid University (ABBU) Tlemcen, Algeria in 2001. His master thesis was the development of an autonomous mobile robot for academic research. His current research interests are control systems and design optimization of VTOL flying robots.

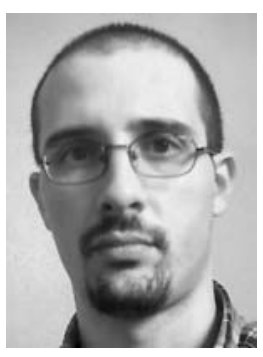

Pierpaolo Murrieri is a Ph.D. student at the "Centro Interdipartimentale E. Piaggio" and "Dipartimento Sistemi Elettrici ed Automazione" (DSEA) at the University of Pisa. He got his Master in Electrical Engineer from University of Pisa in 2000. His master thesis was about the registration of biomedical images. His current research interests are mobile robotics, nonlinear control and artificial vision.

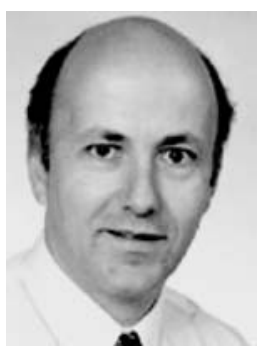

Roland Siegwart is director of the Autonomous Systems Lab (ASL) at the Swiss Federal Institute of Technology Lausanne (EPFL). He received his Masters in Mechanical Engineering in 1983 and his Ph.D. in 1989 at the Swiss Federal Institute of Technology Zurich (ETH). In 1989/90 he spent one year as postdoc at Stanford University. From 1991 to 1996 he worked part time as R\&D director at MECOS Traxler AG and as a lecturer and deputy head at the Institute of Robotics, ETH. In 1996 he joined EPFL as full professor where he is working in robotics and mechatronics, namely mobile robot navigation, space robotics, human-robot interaction, all terrain locomotion and micro-robotics. Roland Siegwart is member of various scientific committees and cofounder of several spin-off companies. 